\section{ORIGINAL RESEARCH}

\author{
P. Mordasini \\ J. Gralla \\ D.-D. Do \\ J. Schmidli \\ B. Keserü \\ M. Arnold \\ U. Fischer \\ G. Schroth
}

C. Brekenfeld

\title{
Percutaneous and Open Retrograde Endovascular Stenting of Symptomatic High-Grade Innominate Artery Stenosis: Technique and Follow-Up
}

\begin{abstract}
BACKGROUND AND PURPOSE: Angioplasty and stenting of the IA have been reported with high technical and clinical success rates, low complication rates and good mid-term patency rates. Different antegrade or retrograde endovascular catheter-based approaches and combinations with surgical exposure of the CCA are used. The purpose of this study was to determine safety, efficacy and mid-term clinical and radiological outcome of the stent-assisted treatment of atherosclerotic stenotic disease of the IA with special focus on the different technical approaches.
\end{abstract}

MATERIALS AND METHODS: Between 1996 and 2008, 18 patients (12 men, 6 women) with symptomatic high-grade stenosis ( $>80 \%$ ) of the IA were treated with endovascular stent placement. Their mean age was 60.4 years (range, 48-78 years). Mean angiographic and clinical follow-up was 2.7 years (range, 0.3-9.1 years). Clinical follow-up was performed by using the mRS at hospital discharge, routine follow-up controls, and a questionnaire. In 11 patients, a percutaneous approach was used. In 7 patients, the lesions were accessed retrogradely through a cervical cut-down with common carotid arteriotomy. In 2 patients, a simultaneous ipsilateral carotid endarterectomy was performed.

RESULTS: In all patients, primary stent placement was performed. There were 2 procedure-related transient complications $(11.1 \%)$ due to cerebral embolism without permanent morbidity or mortality. During the follow-up, all patients showed improvement of the preprocedural symptoms. At the latest clinical follow-up (mean, 2.7 years), all patients showed an excellent or good outcome (mRS, 0 or 1). In 2 patients $(11.1 \%)$, a secondary stent placement was needed due to a significant symptomatic in-stent stenosis.

CoNCLUSIONS: Percutaneous and open retrograde stenting of high-grade stenosis of the IA is a viable less invasive alternatives to open bypass surgery with good midterm clinical results and patency rates.

ABBREVIATIONS: $\mathrm{CCA}=$ common carotid artery; $\mathrm{CEA}=$ carotid endarterectomy; $\mathrm{CTA}=\mathrm{CT}$ angiography; DSA = digital subtraction angiography; $\mathrm{DWI}=$ diffusion-weighted imaging; $\mid \mathrm{A}=$ innominate artery; MRA = MR angiography; $\mathrm{mRS}=$ modified Rankin Scale; PTA = percutaneous transarterial angioplasty; SCA = subclavian artery

A ngioplasty and stent placement in symptomatic atherosclerotic stenoses of the supra-aortic vessels, including the IA, have evolved as effective and safe treatment techniques since the 1990s. ${ }^{1,2}$ Standard open surgical procedures are now mostly reserved for extensive multivessel involvement or after failed endovascular procedures.

Indications for treatment include clinical symptoms ranging from transient ischemic attacks, stroke, subclavian steal syndrome, and vertebrobasilar insufficiency syndromes to upper limb ischemia.

Treatment options include in situ or extra-anatomic bypass surgery, endarterectomy, or endovascular treatment, including balloon angioplasty and/or stent placement. Different antegrade or retrograde endovascular catheter-based approaches and combinations with surgical exposure of the CCA are used. For IA stenosis with concomitant disease of the ca-

Received August 24, 2010; accepted after revision January 24, 2011.

From the Institute of Diagnostic and Interventional Neuroradiology (P.M., J.G., G.S., C.B.), and the Departments of Angiology (D.-D.D.), Vascular Surgery (J.S.), and Neurology (B.K., M.A., U.F.), University of Bern, Bern, Switzerland.

Please address correspondence to Gerhard Schroth, MD, Institute of Interventional and Diagnostic Neuroradiology, Inselspital-University of Bern, Freiburgstr 10, CH-3010 BernSwitzerland; e-mail: gerhard.schroth@insel.ch

Indicates article with supplemental on-line table.

http://dx.doi.org/10.3174/ajnr.A2598 rotid bifurcation, open retrograde angioplasty and stent placement with or without concomitant carotid endarterectomy have been advocated as less invasive treatment alternatives to the traditional surgical procedures. ${ }^{1,3-5}$ Often the IA represents the most challenging supra-aortic trunk vessel due to its large diameter and short length as well as the bifurcation pattern into the right CCA and SCA. Endovascular stent-placement interventions have been reported with high technical and clinical success rates, low complication rates, and good midterm patency rates. ${ }^{1,2,6-10}$ However, potential embolic complications during balloon inflation and stent deployment as well as restenosis remain an important concern.

The purpose of this study was to determine the safety, efficacy, and midterm clinical and radiologic outcome of the stent-assisted treatment of atherosclerotic stenotic disease of the IA, with special focus on the different technical approaches.

\section{Materials and Methods}

\section{Patient Population}

Between 1996 and 2008, percutaneous or open retrograde stent placement in the IA was performed in 18 patients ( 12 men, 6 women) from 48 to 78 years of age (mean, 60.4 years) with high-grade $(>80 \%)$ symptomatic stenosis. Patients were selected for endovascular or combined surgical/endovascular treatment after discussion by an interdisciplinary neurovascular board consisting of attending neurora- 


\begin{tabular}{|c|c|c|}
\hline \multicolumn{3}{|l|}{ Table: Patient characteristics } \\
\hline Parameters & $\begin{array}{c}\text { No of } \\
\text { Patients }\end{array}$ & $\%$ \\
\hline Total No. of patients & 18 & 100 \\
\hline Men & 12 & 66.7 \\
\hline Women & 6 & 33.3 \\
\hline Age (range) (mean) (yr) & $48-78(60.4)$ & \\
\hline \multicolumn{3}{|l|}{ Symptoms } \\
\hline Subclavian steal & 9 & 50.0 \\
\hline Transient ischemic attack & 10 & 55.6 \\
\hline Syncope or presyncope episodes & 2 & 11.1 \\
\hline Symptoms of upper limb ischemia & 1 & 5.6 \\
\hline \multicolumn{3}{|l|}{ Cardiovascular risk factors and comorbidities } \\
\hline Arterial hypertension & 14 & 77.8 \\
\hline Diabetes mellitus & 3 & 16.7 \\
\hline Smoking & 17 & 94.4 \\
\hline Hyperlipidemia & 14 & 77.8 \\
\hline $\begin{array}{l}\text { Coronary heart disease or previous } \\
\text { myocardial infarction }\end{array}$ & 7 & 38.9 \\
\hline Previous aortocoronary bypass surgery & 4 & 22.2 \\
\hline $\begin{array}{l}\text { Previous ischemic stroke or transient } \\
\text { ischemic attack }\end{array}$ & 11 & 61.1 \\
\hline Associated stenosis of other cervical vessels & 14 & 77.8 \\
\hline Peripheral arterial occlusive disease & 9 & 50.0 \\
\hline
\end{tabular}

diologists, neurologists, vascular surgeons, and angiologists. All interventions were part of routine clinical management. Patient data, technical and clinical success, complications of the procedure, and results of follow-up controls were reviewed retrospectively from the patients' charts and radiologic reports. The patients' characteristics, clinical symptoms, cardiovascular risk factors, and comorbidities are summarized in the Table.

All patients underwent preinterventional clinical examination with bilateral arm pressure measurements and duplex sonography. As part of the preoperative work-up, patients underwent either MRA, CTA, or conventional DSA to confirm the suspected lesion.

\section{Technique}

PTA and stent placement were performed with the patient under local anesthesia, whereas open retrograde stent placement with the patient under general anesthesia was performed in the angiography suite on a biplane high-resolution angiography system (CAS 500, Toshiba Medical Systems, Tokyo, Japan until 2007; Artis zee biplane, Siemens, Erlangen, Germany since 2008).

Access to the lesion was gained through a percutaneous puncture of the common femoral artery in 13 patients, of the brachial artery in 1 patient with Leriche syndrome, and by using both accesses in 1 patient with a residual stenosis for postdilation of the stent in a second session. Retrograde access through surgical exposure of the right CCA was performed in 7 patients. Technical characteristics of the interventions are summarized in On-line Table 1.

Using the antegrade femoral approach, we obtained angiographic images by using either a $5 \mathrm{~F}$ diagnostic catheter proximal to the IA origin or a pigtail catheter placed in the ascending aorta to define the exact location and severity of the lesion.

Then an $8 \mathrm{~F}$ guiding catheter or a long 6F-7F sheath (SuperArrow -Flex PSI Set; Arrow International, Reading, Pennsylvania) was used, depending on operator preference, lesion location, and aortic arch configuration. For the brachial approach, a long 6F sheath was used. Guidewires measuring $0.018-0.035$ inches were used, depending on operator choice. Whenever possible, the procedure was completed via the femoral approach because of better visualization and easier stent deployment. In patients with a combined brachial and femoral approach, the femoral catheter was placed in the aorta or in the proximal IA with its tip pointing to the stenotic site.

Through the transbrachial approach, a 0.035 -inch $(260 \mathrm{~cm}$ long) guidewire (Radiofocus Guide Wire; Terumo, Tokyo, Japan) was navigated retrogradely into the descending aorta. Then by using a snare, the wire was lead through the femoral sheath, resulting in a wire through the whole body from the brachial to the femoral artery. This procedure allows the introduction of a smaller sheath for brachial access. Retrograde stent placement was then attempted from the brachial access by using a stiff hydrophilic guidewire.

To protect against embolic events in the right vertebral artery during open and endovascular stent placement, we inflated a pressure cuff bilaterally, exceeding the systolic arterial pressure on the left and right upper arms. This procedure temporarily increases retrograde flow in the right vertebral artery. Then the right pressure cuff was deflated to further increase flow reversal in the right vertebral artery by the induced hyperemia in the right upper limb. ${ }^{11}$

\section{Hybrid Therapy}

The hybrid approach by using an open and an endovascular technique with surgical exposure of the CCA with distal clamping to prevent cerebral embolization before retrograde stent deployment was used in 7 patients.

Surgical access to the CCA was described previously. ${ }^{12,13}$ Briefly, a standard cervical approach to the carotid bifurcation by using an incision along the sternocleidomastoid muscle with the patient under general anesthesia was performed in the angiography suite. Retrograde puncture of the exposed CCA by using a 16-ga needle was followed by the insertion of a short $6 \mathrm{~F}$ sheath. A roadmap of the target lesion was acquired either by using the transfemorally introduced diagnostic 5F guiding catheter placed with the tip in the prestenotic segment of the IA (5F, JB 2) or in the ascending aorta (pigtail). Clamping of the distal CCA to prevent cerebral embolization was then performed just before retrograde crossing of the lesion with a 0.035 -inch guidewire as well as during stent deployment. Opening of the side branch of the CCA sheath allowed drainage of potential debris before restoration of the carotid flow by removing the clamp and flushing the CCA with a heparinized solution to avoid thrombus formation. The technical success of the treatment was controlled by antegrade and retrograde angiography. The arteriotomy was closed by using a $6-0$ Prolene suture (Ethicon, Cincinnati, Ohio), and the clamp was removed.

In 2 patients with concomitant high-grade stenoses of the internal carotid artery, a combined procedure consisting of carotid endarterectomy with a bovine pericardial patch reconstruction of the carotid bifurcation and subsequent retrograde stent placement in the IA was performed.

\section{Stent Placement}

Stent selection was based on vessel reference diameter and lesion length. Stainless balloon-expandable stents were preferred due to their better delivery profile and deployment precision. The stent diameter was chosen not to exceed the vessel reference diameter. Twenty lesions were treated, including 2 secondary procedures. Due to the long 12-year period of patient inclusion, we used various stents: Palmaz stent (Cordis Corporation, Miami Lakes, Florida) in 4, WaveMax (Jomed, Benningen, Switzerland) in 8, Jostent (Abbott Laboratories, Abbott Park, Illinois) in 1, Easy Wall stent (Boston Scientific, Galway, Ireland) in 1, Bridge stent (Medtronic AVE, Düsseldorf, Ger- 

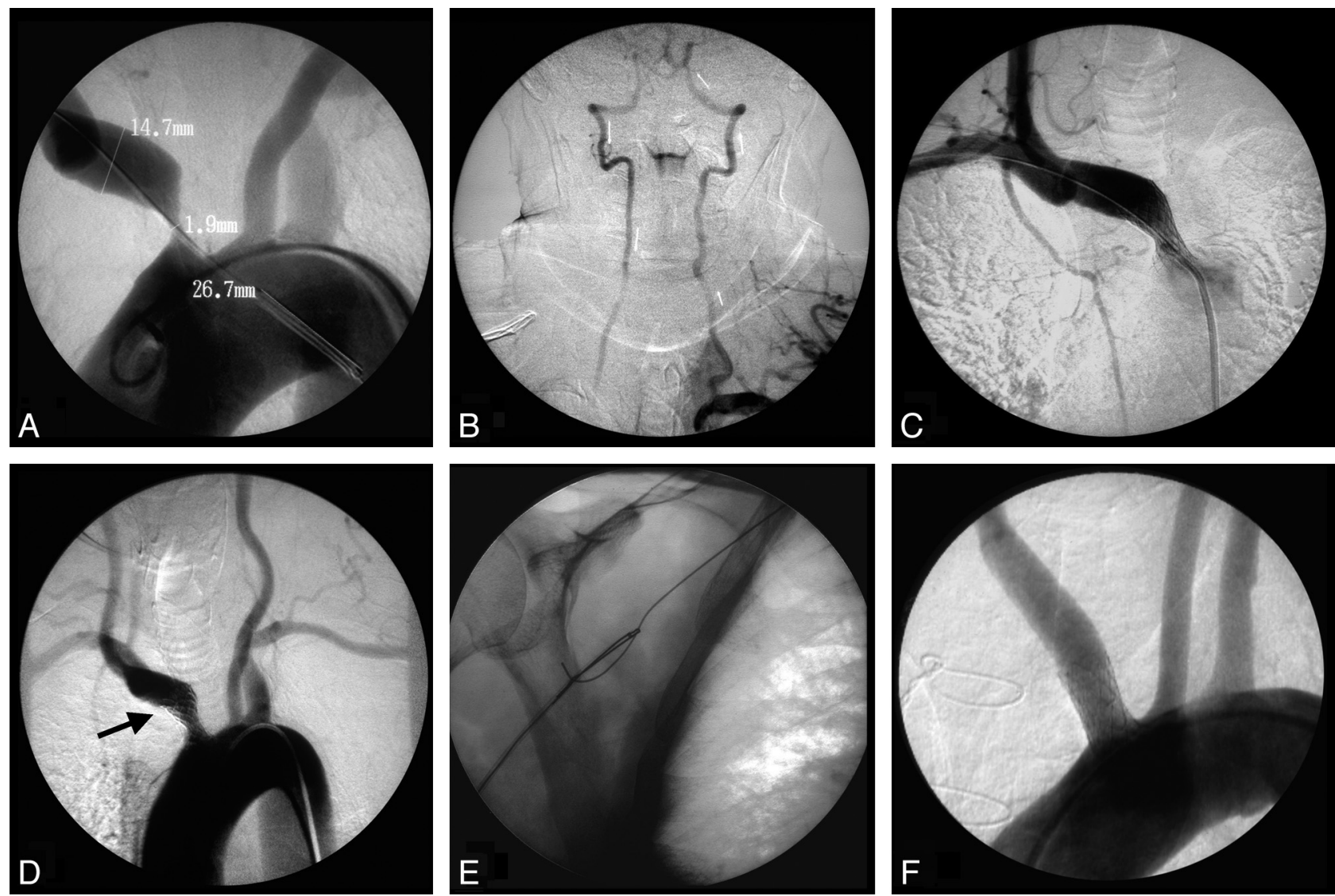

Fig 1. Case 1. $A$ and $B$, Aortic arch DSA showing a high-grade stenosis of the IA in a 64-year-old patient and demonstrating a vertebrovertebral steal with retrograde flow in the right vertebral artery (arrows). C, Antegrade stent placement (Palmaz, $12 / 3 \mathrm{~cm}$ ) and balloon dilation of the stenosis. $D$, Control angiogram shows a residual stenosis at the proximal $\mid \mathrm{A}$ and slightly nonadherent stent meshes (arrow) in the distal part. With a transbrachial approach, a 0.035 -inch-long $(260 \mathrm{~cm})$ guidewire (Terumo) was navigated retrogradely through the stent into the descending aorta. $E$, With a snare, the wire was lead through the femoral sheath, resulting in a wire through the whole body from the femoral to brachial artery. $F$, Introduction of a balloon catheter retrogradely through the brachial end of the wire and postdilation of the stenosis, resulting in no residual stenosis and completely adherent stent meshes.

many) in 2, Herculink stent (Abbott Laboratories) in 1, Invastent Onda (Invatec, Roncadelle, Italy) in 1, Omnilink stent (Abbott Laboratories) in 1, and Scuba (Invatec) in 1 lesion. The stent diameter varied from 7 to $12 \mathrm{~mm}$, and the length, from 17 to $30 \mathrm{~mm}$.

After the interventional procedure, a final angiography was always performed to confirm the technical success and patency of the vessel. Technical success was defined as a stenosis grade reduction of $>80 \%$ of the target vessel. In the percutaneously treated patients, no embolic protection device was used.

\section{Pharmacologic Treatment}

Aspirin, $100 \mathrm{mg}$ daily, was given for at least 5 days before the intended procedure and was continued afterward on a life-long basis. In 9 pa-tients, clopidogrel, $75 \mathrm{mg}$ daily, was added for 6 months. During the procedure, 5000-6000 IU of heparin was administered.

\section{Follow-Up}

Clinical follow-up consisted of a history of recurrent or new onset of symptoms, physical examination including peripheral pulses, bilateral arm blood pressure measurement, and duplex sonography postinterventionally and at 3,6, and 12 months. MRA or DSA was indicated if significant change in the duplex examination or relevant clinical symptoms occurred and if duplex sonography was not conclusive or if additional new or progressive occlusive lesions of the supra-aortic vessels were suspected. For midterm follow-up, we requested clinical examination, duplex sonography, and MRA for all patients. Clinical outcome was assessed by using the $\mathrm{mRS}$ at hospital discharge, routine follow-up controls, and a questionnaire for midterm follow-up.

\section{Results}

\section{Technical Success}

Stent deployment could be achieved in all patients (100\%). Technical success was $94.4 \%$ (17/18). In 1 patient, the distal part of the stent was not completely apposite to the predilatated vessel wall. This outcome resulted in a 50\%-75\% residual stenosis. Balloon postdilation within the stent in a second session 2 days after the initial treatment resulted in complete apposition of the stent meshes at the vessel wall without residual stenosis (Fig 1).

\section{Complications}

There were 2 cases $(11.1 \%)$ of procedure-related transient complications due to cerebral embolism. In both patients, endovascular PTA/stent placement was performed without an embolic protection device: One patient had a transient lefthand weakness with a diffusion restriction depicted by cranial MR imaging in the right hand notch, which resolved after 24 hours. Postinterventionally, 1 patient had a neglect and a fluctuating left hemiparesis with complete recovery after 24 hours. Cranial MR imaging showed small embolic ischemic lesions in 
the right caudate nucleus and the precentral and occipital regions.

In 2 patients, clinically silent diffusion restrictions could be detected by postinterventional MR imaging after PTA/ stent placement. There were no embolic complications among the patients who underwent open retrograde stent placement. There was no procedure-related permanent morbidity or mortality. No access-related complication occurred.

\section{Follow-Up and Restenosis}

Follow-up varied between 0.3 and 9.1 years (mean, 2.7 years). At least 1 clinical and radiologic follow-up could be performed in all 18 patients. Immediate complete resolution or improvement of the vertebrobasilar steal syndromes (subclavian steal, upper limb ischemia, syncope/presyncope) could be achieved in all patients. In patients with asymmetry in arterial blood pressure in the upper limbs with a gradient exceeding $20 \mathrm{~mm}$ $\mathrm{Hg}$, a normalization could be achieved. At the latest clinical follow-up, all patients showed an excellent or good clinical outcome according to an mRS score of 0 or 1 .

In 2 patients $(11.1 \%)$, restenting due to a symptomatic restenosis of the lesion was needed. One patient had another transient monocular blindness 10 months after endovascular PTA/stent placement. Control duplex sonography showed a midgrade concentric restenosis of the proximal stent with an incomplete steal phenomenon. Annual follow-up was performed, and after 34 months, a progression to a high-grade stenosis was detected. The patient underwent endovascular restenting with a Jostent (Abbott Laboratories). One patient developed signs of upper limb ischemia and transient monocular blindness 10 months after open retrograde stent placement. A short high-grade proximal restenosis was found at follow-up sonography. The patient underwent restenting with a Palmaz stent (Cordis) (Fig 2). In all other patients, no significant restenosis was found at latest follow-up.

\section{Discussion}

Symptomatic stenotic disease of the IA is uncommon compared with stenosis of other supra-aortic trunk vessels. Endovascular treatment of the IA is often challenging due to its large diameter, short length, and the proximity to the origin of the right CCA. Different approaches and methods have been described by various groups, ranging from minimally invasive percutaneous endovascular angioplasty and stent placement to open, hybrid, and combined approaches. However, the literature on the endovascular or hybrid open treatment of the IA is limited and consists only of small series with short clinical follow-ups. ${ }^{1,6-10}$ Furthermore, most of the series did not focus on the treatment of IA stenosis alone but combined the outcomes of the treatment of vertebral, subclavian, and carotid arteries. $^{2,14-20}$ Therefore, to our knowledge, this series consisting of 18 consecutive patients, is so far one of the largest cohorts focused on stent placement procedures for high-grade IA stenosis available in the literature.

In a meta-analysis performed by Hadjipetrou et $\mathrm{al}^{2}{ }^{2}$ evaluating the open surgical outcome in subclavian and brachiocephalic artery obstruction in $>2000$ patients, the mortality rate was $3 \%$, the stroke rate was $2 \%$, and the overall complication rate was $16 \%$.

The 6 most recent studies about endovascular treatment of IA stenotic disease included 51 patients, with a maximum of 10 patients included in 2 studies. ${ }^{1,6-10}$ The authors reported technical success rates of $100 \%$, no periprocedural mortality, only 1 case of a transient neurologic deficit, and 1 access-related complication consisting of a brachial artery thrombosis, which required thrombectomy. The patency rates ranged from $78 \%$ to $100 \%$ within $12-35$ months of follow-up.

Palchik et $\mathrm{al}^{10}$ compared the role of endovascular and open surgical approaches in the treatment of symptomatic IA stenosis in 18 patients, 10 patients in the endovascular and 8 patients in the surgical group. There were no thromboembolic events except 1 access-related complication (1/10) in the endovascular group and 2 systemic pulmonary complications in the surgical group (2/8). Patency rates were $78 \%$ in the endovascular group after 25 months and $80 \%$ in the surgical group after 27 months' follow-up. Restenosis occurred in 1 patient after 34 months in the endovascular group and in 1 patient in the surgical group; 1 additional patient had an asymptomatic bypass graft thrombosis.

Hybrid, open, and combined endovascular techniques with exposure of the CCA with distal clamping to prevent distal embolization before retrograde stent deployment were described by Queral and Criado in 1996, ${ }^{1}$ with no patients having perioperative strokes.

Recognizing the importance of cerebral protection against thromboembolic events, Peterson et $\mathrm{al}^{8}$ reported their experience in aortic arch vessel stent placement, including 8 IA stenoses by using cerebral protection by either open surgical distal CCA clamping or endovascular protection devices without mortality or complications. In 1 case of IA stenosis, cerebral protection was not possible due to a brachial approach. Payne et $\mathrm{al}^{5}$ reported no new neurologic deficits in their study of cerebral protection during open retrograde angioplasty/stent placement of 4 IA lesions, including a synchronous carotid endarterectomy in 1 case. During the angioplasty and a stent placement procedure, no emboli were detected in the ipsilateral middle cerebral artery territory by using transcranial Doppler monitoring.

In our series, there were 2 transient neurologic deficits in the endovascular group (2/11), without a permanent neurologic deficit. In 2 patients, clinically silent ischemic lesions could be found by postinterventional DWI examination. Therefore, thromboembolic complications may be a concern, especially in highly calcified or more extensive lesions. In the open retrograde group, there was no periprocedural neurologic deficit, indicating that distal clamping of the CCA is an effective protection against procedural embolic events. On the other hand, there were no access-related complications, underlining the minimally invasive character of the interventions.

Our strategy of treating IA lesions is mainly based on the following points: degree of stenosis, amount of calcified plaques at the ostium and at the site of stenosis, and IA branching with respect to the anatomy of the aortic arch.

Selective catheterization of the IA is challenging in cases with branching of the IA from the aortic arch in a steep angle (eg, bovine type 3 configuration of the aortic arch), a 

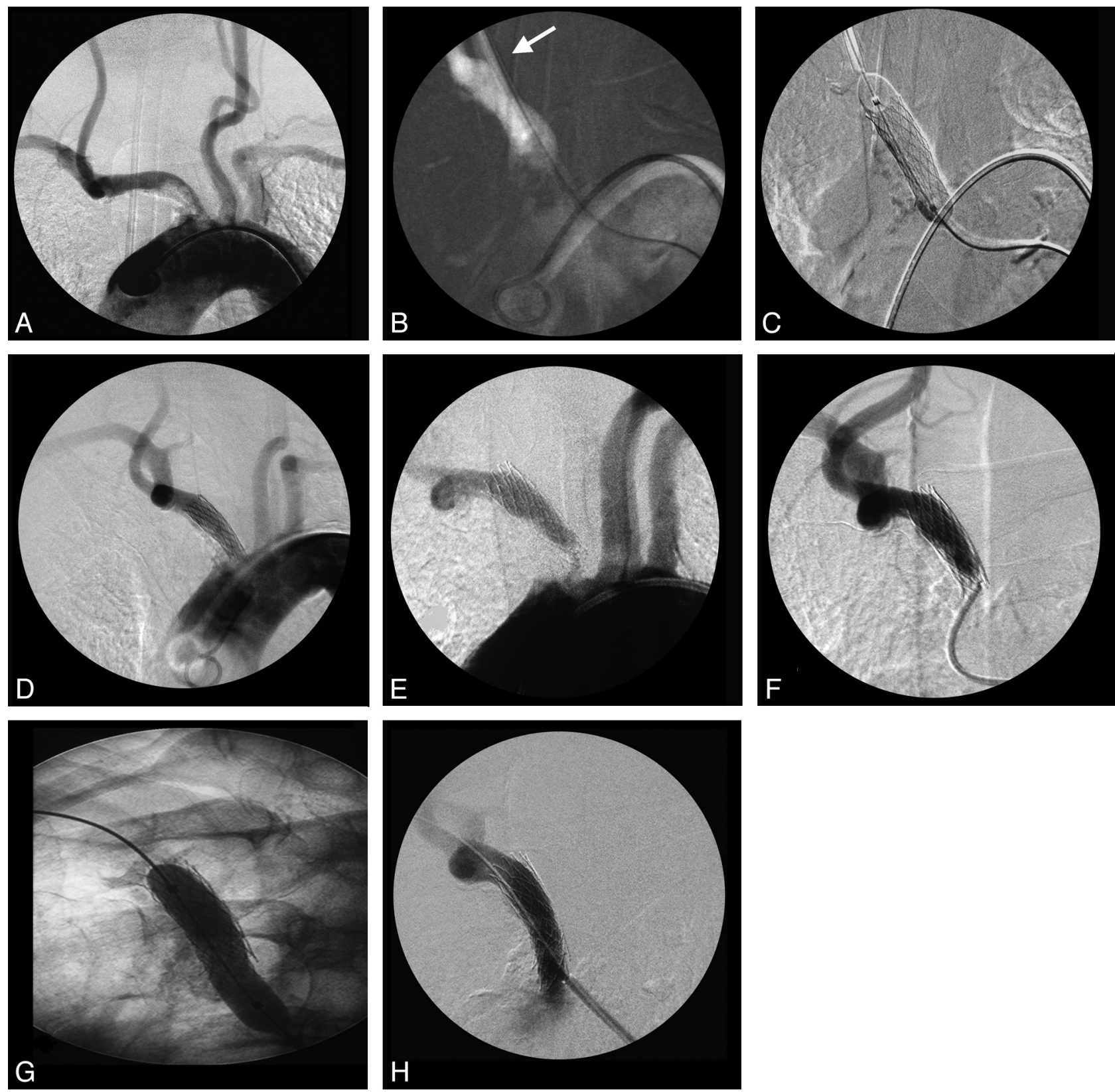

Fig 2. Case 2. A, Aortic arch DSA through a pigtail catheter in the aortic arch introduced by a femoral approach in a 74-year-old patient with several TIAs and transient monocular blindness, depicting a long high-grade IA stenosis with irregularly configurated circumferential plaque. $B$, Retrograde introduction of an $8 \mathrm{~F}$ sheath (arrow) and passing of the lesion by using a 0.035 -inch guidewire (Terumo) guided by a roadmap of the aortic arch through the diagnostic pigtail catheter. $C$, Predilation of the lesion by using a balloon catheter and introduction and deployment of the balloon-mounted stent (Palmaz, 10/30 mm). D, Postinterventional control angiogram shows no residual stenosis and correctly placed stent meshes at the origin of the IA. $E$, One year after the treatment, the patient again had transient monocular blindness and a weakness of the right arm. $F$, Control angiography shows a high-grade restenosis of the proximal stent at the origin of the IA. $G$ and $H$, Predilation of the stenosis by using a balloon catheter and restenting by using in-stent deployment of a Palmaz stent over a $9 F$ guiding catheter.

high load of arteriosclerotic plaques at the ostium of the IA, and in high-grade near-occlusive lesions. These circumstances may require repetitive catheterization attempts from an unstable position of the transfemoral catheter in the aortic arch, increasing the risk of embolic events. In these cases, we learned that a retrograde transbrachial or open surgical approach combined with a femoral approach is useful and facilitates the procedure. Passing the lesion retrogradely with a wire is easier to perform because we avoid navigation over a long distance without much stability to pass a high-grade filiform stenosis. Capturing the wire in the descending aorta and leading it out through a femoral access allows a second step to stent the lesion less traumatically, either antegradely from the femoral approach or retrogradely through the brachial approach as described in patient 1 . The resulting wire across the whole body from the brachial to femoral artery allows stretching of the angulated branching of the IA from the aortic arch and stretching of the angle of the stenotic segment in the direction of the SCA, leading to a more stable position for stent deployment.

Therefore, in our view, the most important decision points in favor of a retrograde approach and against an exclusive antegrade femoral approach are the degree of aortic arch arte- 
riosclerotic lesions around the ostium of the IA, the degree of stenosis, and a steep angulation of the IA from the aortic arch.

Furthermore, in our experience, the use of a stent as short as possible without undersizing is paramount to avoid stent migration during deployment and to allow precise stent placement in relation to the ostium of the IA, avoiding stent extension into the aortic arch. The use of the utmost shortest stent length decreases the risk of kinking and fracture of the stent mesh as well as the risk of in-stent thrombosis and stenosis. However, precise placement of a short stent requires a stable situation, which can be obtained with a wire from the femoral to the brachial sheath, which can be stretched during deployment of the stent.

In cases with high-grade tandem stenosis of the IA and the carotid bifurcation, it is sometimes difficult to define the symptomatic lesion or whether the possible additive effect of a tandem stenosis of the IA and the carotid artery on perfusion is an additional effect of both lesions, resulting in either embolic or hemodynamic symptoms. Simultaneous treatment of both lesions, consisting of a carotid endarterectomy combined with retrograde stent placement of the tandem lesion in the IA, has been described by various groups. ${ }^{3-5,21,22}$ Allie et $\mathrm{al}^{4}$ reported the combined treatment in 11 IA lesions, with a procedural success rate of $97 \%$, and no significant restenosis found during a 34-month follow-up.

\section{Conclusions}

Percutaneous endovascular and retrograde open stent placement in high-grade stenoses of the IA is a viable alternative to open bypass surgery, with good clinical results and high patency rates. Thromboembolic complications, even if silent, still remain a matter of concern, and embolic protection may be needed.

Disclosures: Gerhard Schroth, Research Support (including provision of equipment or materials): University Hospital.

\section{References}

1. Queral LA, Criado FJ. The treatment of focal aortic arch branch lesions with Palmaz stents. J Vasc Surg 1996;23:368-75

2. Hadjipetrou P, Cox S, Piemonte T, et al. Percutaneous revascularization of atherosclerotic obstruction of aortic arch vessels. J Am Coll Cardiol 1999;33: $1238-45$

3. Grego F, Frigatti P, Lepidi S, et al. Synchronous carotid endarterectomy and retrograde endovascular treatment of brachiocephalic or common carotid artery stenosis. Eur J Vasc Endovasc Surg 2003;26:392-95

4. Allie DE, Hebert CJ, Lirtzman MD, et al. Intraoperative innominate and common carotid intervention combined with carotid endarterectomy: a "true" endovascular surgical approach. J Endovasc Ther 2004;11:258-62

5. Payne DA, Hayes PD, Bolia A, et al. Cerebral protection during open retrograde angioplasty/stenting of common carotid and innominate artery stenoses. Br J Surg 2006;93:187-90

6. Brountzos EN, Petersen B, Binkert C, et al. Primary stenting of subclavian and innominate artery occlusive disease: a single center's experience. Cardiovasc Intervent Radiol 2004;27:616-23

7. Sullivan TM, Gray BH, Bacharach JM, et al. Angioplasty and primary stenting of the subclavian, innominate, and common carotid arteries in 83 patients. $J$ Vasc Surg 1998;28:1059-65

8. Peterson BG, Resnick SA, Morasch MD, et al. Aortic arch vessel stenting: a single-center experience using cerebral protection. Arch Surg 2006;141: $560-63$

9. Ruebben A, Tettoni S, Muratore P, et al. Feasibility of intraoperative balloon angioplasty and additional stent placement of isolated stenosis of the brachiocephalic trunk. J Thorac Cardiovasc Surg 1998;115:1316-20

10. Palchik E, Bakken AM, Wolford HY, et al. Evolving strategies in treatment of isolated symptomatic innominate artery disease. Vasc Endovascular Surg 2008; 42:440-40. Epub 2008 Jul 11

11. Kashyap VS, Franklin KS, Fitzpatrick CM. Bilateral brachial artery occlusion decreases internal carotid artery volume flow: a simple adjunct for cerebral protection? J Endovasc Ther 2005;12:454-60

12. Lutz HJ, Do DD, Schroth G, et al. Hybrid therapy of symptomatic stenosis of the innominate artery. Eur J Vasc Endovasc Surg 2002;24:184-85

13. Schroth G, Do DD, Remonda L, et al. Special techniques for angioplasty of the brachiocephalic vessels [in German]. Rofo 1997;167:165-73

14. Zaytsev AY, Stoyda AY, Smirnov VE, et al. Endovascular treatment of supraaortic extracranial stenoses in patients with vertebrobasilar insufficiency symptoms. Cardiovasc Intervent Radiol 2006;29:731-38

15. Woo EY, Fairman RM, Velazquez OC, et al. Endovascular therapy of symptomatic innominate-subclavian arterial occlusive lesions. Vasc Endovascular Surg 2006;40:27-33

16. Harada K, Nakahara I, Tanaka M, et al. Therapeutic strategy and outcome of stenting for subclavian and innominate artery occlusive disease [in Japanese]. No Shinkei Geka 2004;32:151-58

17. Schroth G, Remonda L, Dai DD, et al. Stents in the treatment of supraaortic vessel stenosis [on German]. Ther Umsch 2003;60:190-98

18. Gonzalez A, Gil-Peralta A, Gonzalez-Marcos JR, et al. Angioplasty and stenting for total symptomatic atherosclerotic occlusion of the subclavian or innominate arteries. Cerebrovasc Dis 2002;13:107-13

19. Phatouros CC, Higashida RT, Malek AM, et al. Endovascular treatment of noncarotid extracranial cerebrovascular disease. Neurosurg Clin $\mathrm{N} \mathrm{Am}$ 2000;11:331-50

20. Azzarone M, Cento M, Mazzei M, et al. Symptomatic subtotal occlusion of the innominate artery treated with balloon angioplasty and stenting. J Endovasc Ther 2000;7:161-64

21. Macierewicz J, Armon MP, Cleveland TJ, et al. Carotid endarterectomy combined with proximal stenting for multilevel disease. Eur J Vasc Endovasc Surg 2000;20:572-75

22. Arko FR, Buckley CJ, Lee SD, et al. Combined carotid endarterectomy with transluminal angioplasty and primary stenting of the supra-aortic vessels. J Cardiovasc Surg (Torino) 2000;41:737-42 